\title{
Coal Mine Gas Inspecting System Based on Data Fusion of Multi-Sensor
}

\author{
Zhifu Gao \\ School of Electronics and Information Engineering \\ Anhui University \\ Hefei, 230601, China \\ llyahu@163.com
}

Linsheng Huang

School of Electronics and Information Engineering Anhui University

Hefei, 230601, China

linsheng0808@163.com

\author{
Dan Wang \\ School of Electronics and Information Engineering \\ Anhui University \\ Hefei, 230601, China \\ 18297955876@163.com
}

\begin{abstract}
This paper illustrates a low energy consumption and high accuracy WSN (wireless sensor network) that provides an intelligent gas inspecting system in coal mines, which offsets the shortage of mine cable monitoring system. The substation of coal mine gas inspecting system was designed by $\mathrm{CC2530}$ SOC that is responsive for data acquisition and transmission, which integrates sensor technology, wireless communication technology and data fusion technology. The overall framework of the system was given, and the WSN was designed in detail, including gas sensor, inspecting substation. The system offers the real-time data transmission between underground mine and surface monitoring centre through highly secure, reliable wireless sensor network, which improves the mine's safety.
\end{abstract}

Keywords- Coal Mine Gas; Inspecting System; MultiSensor; Data fusion; WSN

\section{INTRODUCTION}

Coal mine accidents were unforeseen and they are caused by many factors, which not only bring huge economic losses, but also pose a great threat to the safety of human life [2]. It has great significance to guarantee the safety of underground mine operations, installation of environmental inspecting devices in the roadway to supervise environmental parameters. However, the traditional detecting methods generally use the wired connection to communicate with the monitoring centre, which was very difficult and also cost too much to set up the networks [3]. In contrast, the wire less sensor network can be easily deployed with the current network connection of mine, which have good compatibility, easy to form mine gas monitoring network and suit a variety of different situations [4].

\author{
Chao Xu \\ School of Electronics and Information Engineering \\ Anhui University \\ Hefei, 230601, China \\ xuchao@ adu.edu.cn \\ Qingshan Shi \\ School of Electronics and Information Engineering \\ Anhui University \\ Hefei, 230601, China \\ sqs1277996463@163.com \\ Xiaoming $\mathrm{Wu}$ \\ School of Electronics and Information Engineering \\ Anhui University \\ Hefei, 230601, China \\ 2721822385@qq.com
}

Zigbee is a wireless self-organized network communication protocol which has lots of advantages including low power consumption, low cost, short delay and high capacity. It integrates automatic network protocol, the optimal transmission path selection protocol and Wireless transceiver protocol. Furthermore, Zigbee has strong noise immunity. Therefore, the technology of WSN can not only greatly promote the development of wireless networks, but also raise the accuracy and reliability of transmission data [5].

\section{STRUCTURE OF INSPECTING SYSTEM}

The structure of the mine gas inspecting system based on WSN is shown in Fig. 1. This system mainly consists of the monitoring center, CAN-bus and WSN which includes sensor nodes, router nodes, substation and network gateway. Gas inspecting devices are zigbee wireless sensor network nodes which includes mobile nodes and fixed nodes. The mobile nodes are installed on the mobile devices, such as miner's tools. The fixed nodes, who can acquire the concentration of coal gas, are the routers which recognize the mobile nodes and transmit data between fixed nodes and the mobile nodes. The router is routing node in the WSN, and shares the similar function with the fixed inspecting nodes, except that it doesn't have any sensors which are used to inspect the concentration of coal gas. Monitoring substation can make the appropriate control when the gas concentration exceeds the prescribed limits, which includes the display module, sound and light alarm module and the closed electrical device on the bas is of the router. The network gateway is the network coordinator who serves as a bridge between wireless network and wired network, as it links WSN with the CAN-bus, which is used to exchange the data between 
underground wireless and surface monitoring center, which decodes the data from the CAN-bus and uses sound and light to alarm followed determine the transfinite site according to address code.

The implementation of the topology of the WSN is the main part of the system. When researchers deploy the wireless sensor network nodes, the fixed inspecting nodes and substations should be placed in the appropriate location of the coal face laneway. The router nodes are fixed in the main channels of the district. The gateway of WSN can be

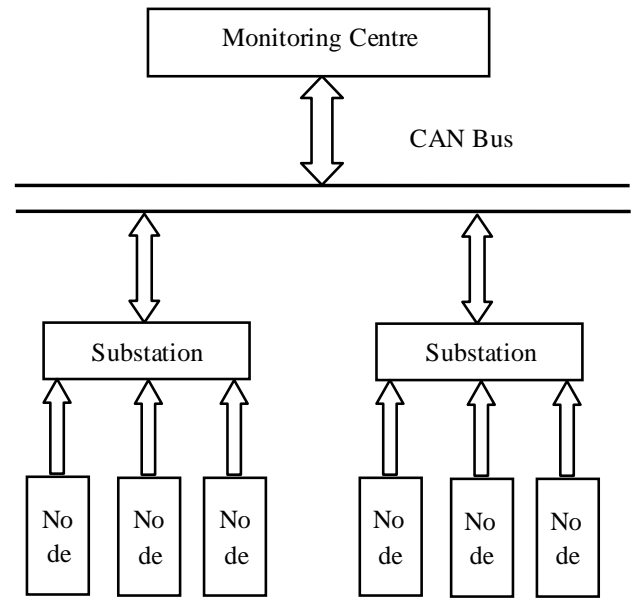

Figure 1. The structure of inspecting system

placed anywhere according to the actual environment of underground coal mine, e.g. positioned in a place of the bottom of the main channel. The data from inspecting nodes through the router nodes are sent to the wireless gateway, which sent the data to the surface monitoring center. The data are acquired by the mobile inspecting nodes in the case of any routers can be identified and forwarded by that router so as to constantly monitor the gas concentration from all directions.

\section{IMPLEMENTATION OF HARDWARE}

The implementation of system hardware mainly contains the design of WSN nodes, such as the inspecting nodes (fixed nodes and mobile nodes), monitoring substation and wireless gateway. The paper chooses CC2530 which includes a microcontroller and a zigbee module as the core chip. The CC2530 produced by Chipcon Company is a true SOC (system-on-chip) solution specifically cut for IEEE802.15.4 and zigbee applications. It enables zigbee nodes to be built with very low gross billof-material consumes. The CC2530 combines the excellent performance of the leading CC2420 RF transceiver with an industry standard enhanced $8051 \mathrm{MCU}, 32 / 64 / 128 \mathrm{~KB}$ flash memory, $8 \mathrm{~KB}$ RAM, analog-digital-converter (ADC), several timers (Timer), AES128 co-processor, watchdog timer (Watchdog Timer), $32 \mathrm{kHz}$ crystal sleep mode timer, power-on reset circuit (Power On Reset), brown-out detection circuit (Brown Out Detection) and 21 programmable $\mathrm{I} / \mathrm{O}$ pins. Combined with the industry leading zigbee protocol stack from Wireless/Chipcon, the CC2530 provides the most promising zigbee solution in the market.

\section{A. Implementation of Inspecting Sensor Nodes}

The inspecting sensor nodes serve as mobile inspecting nodes and fixed inspecting nodes. The latter is fixed in the coal face channels, which not only acquires the gas concentration, temperature and relative humidity, but also serves as the wireless network router to transmit environment data and manage other mobile network nodes. In order to enhance the smooth of the wire less flow and the robustness of the sensor network, its energy supply and battery life should be taken into consideration when the nodes work. Fixed inspecting nodes are made up of multisensors, mic rocontroller, zigbee module and power module, and have relatively simple structure and functions. Data acquired by sensors will be sent to processing module where data are dealt with and then sent by the wireless module.

Mobile sensor nodes are providing for miners and mobile devices, on which the display module and sound and light alarm module are added, and have some advantages in small size, low power consumption and carrying easily. The mobile sensor nodes can be installed on the miner's lamps or mobile tramcar and shearer as well as made into the hand-held gas detector which is used for the gas inspecting workers. The mobile inspecting nodes mainly include sensors, microcontroller, zigbee module, power module, sound and light alarm module and concentration display module. The structure of mobile inspecting node is shown in Fig. 2.

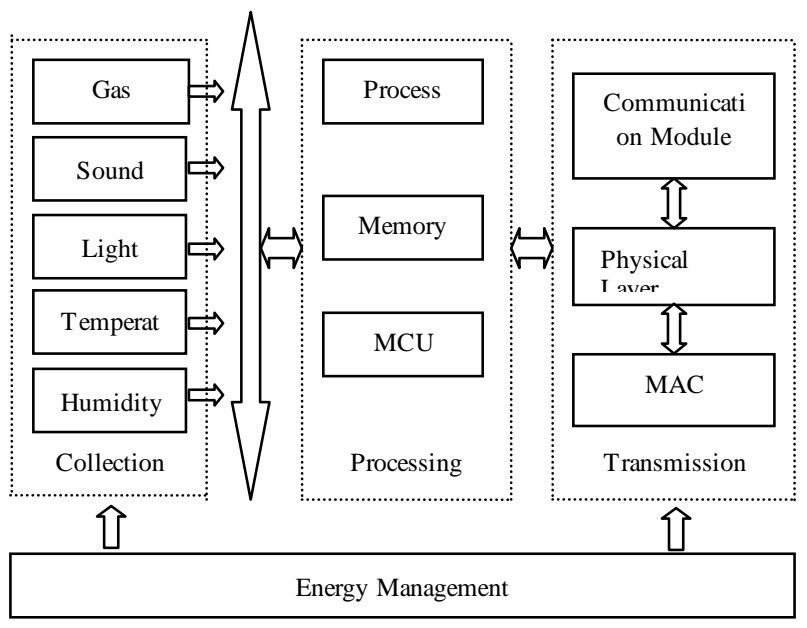

Figure 2. The structure of inspecting sensor nodes

The selection of sensors mainly considers its stability and cost. Due to relatively large demand for the fixed nodes, catalytic combustion methane sensors with stable performance relatively mature technology and low cost is adopted. When mobile node is applied in miner's lamps or mobile cars, catalytic combustion methane sensors are selected; applying them in the hand-held gas inspectors, researchers can use the most advanced intelligent nondispersive infrared methane sensor. Considering the display effectiveness and capabilities of the concentration display module, researchers select digital tube in miner's lamp and other equipments, and select liquid crystal display modules in the hand-held gas detector. In short, mobile detection node is of flexible design, small volu me and is designed according to the actual situation, such as adding or removing certain features. 


\section{B. Implementation of Substation}

The structure of substation mainly contains the CC2530, sound and light alarm module, data collection module, energy management module and display module, as is shown in Fig. 3. Receiving the data, CC2530 will judge and display the data. If the gas concentration exceeds the prescribed limits, the electricity will be cut off by the CC2530 and alarm with sound and light at the same time. On the contrary, the data will be forwarded directly by the CC2530. Since the monitoring sub-station is installed in the vicinity of the coal face, it will achieve the partial power cut for a coal face. The device is provided with power by the AC-DC isolated switching power module. When the AC power outages, the monitoring sub-station will start the DC power (intrinsically safe power) whose supply time should be no less than two hours by the power control module. In the design of monitoring sub-station, nixie tube is used in display module; closed electrical installations involve relay and the corresponding peripheral circuits; DC electromagnetic switch HJR-3FF which uses $5 \mathrm{~V}$ level driver to control the $120 \mathrm{~V}$ to $240 \mathrm{~V}$ AC power is used in relay.

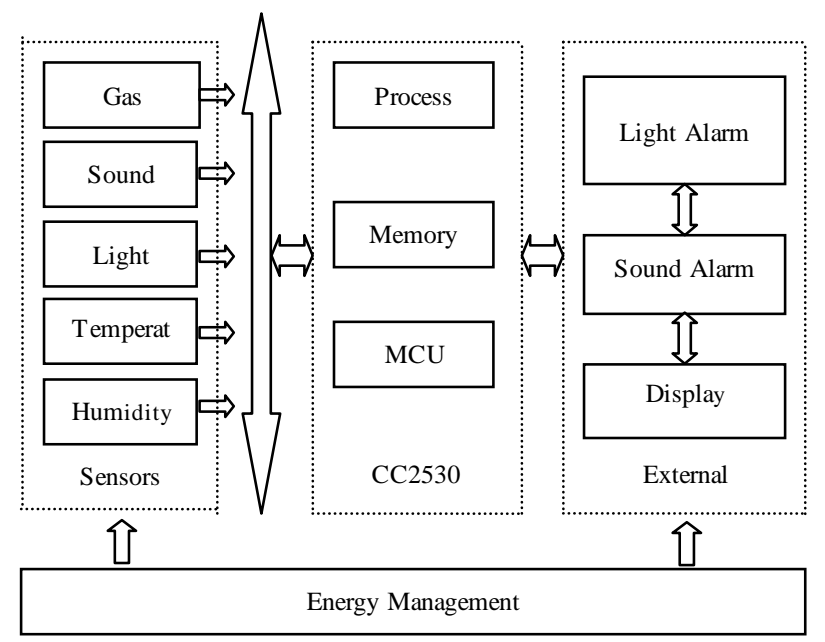

Figure 3. The structure of substation

\section{Implemenation of Wireless Gateway}

The structure of wireless gateway mainly contains the CC2530, sound and light alarm module, closed electrical device, voltage conversion circuit and display module. Received by CC2530, the data will be judged and displayed and send to the CAN module, which complete the communication with the surface monitoring center, after the address code of substation is added. If the gas concentration exceeds the prescribed limits, the electricity will be cut off by the CC2530. At the same time, monitoring gateway will show the transfinite location and alarm with sound and light. The power outage occurs here in a large scale, either in a mining area or even the entire mine. The device's power section uses the same circuit design of monitoring substation. Display module not only shows the concentration but other data and information, so fully functional large-size LCD module is used such as 12864. The CSM100 chip is used by CAN module. The CSM 100 is an embedded CAN to UART chip which integrate the microprocessor, CAN-bus controller, CANbus transceiver, DC-DC module and high-speed optical isolation. It can be easily embedded into devices with a UART interface, and allow devices to access CAN-bus communication interface to achieve the data communication between UART device and CAN-bus network without changing the hardware structure. Closed electrical installation includes relay and the corresponding peripheral circuits. DC electromagnetic switch HJR-3FF is used by the relay.

\section{IMPLEMENTATION OF SOFTWARE}

\section{A. The building of wireless sensor network}

The system allocates zigbee network address by distributed address allocation scheme, and uses cluster tree structure to establish network. Meanwhile, router (including fixed detection node) is used as parent device, and wire less gas sensor is used as child device. Monitoring gateway, as network coordinator, starts network connection, selects a channel, determines a unique PAN address, and broadcasts networking information. Establishing the network, monitoring gateway sets up its own address 0X0000. Router and wireless gas sensor join the network as nodes. Network address involves three parameters, including: the maximum allowed sub-nodes $\mathrm{Cm}$, the maximum allowed router nodes $\mathrm{Rm}$ and the maximum network depth of Lm. According to which researchers can calculate the address interval of neighboring nodes from below each level.

\section{B. The Implementation of Inspecting System Software}

The implementation of inspecting system software consists of wireless sensor nodes, substations, wireless gateway and ground monitoring center's design. The first three parts are zigbee network elements. The CC2430 is a system-on-chip (soc) solution based on zigbee technology, and it has specialized hardware and software development platform. In this design, the integrated development environment of IAR and zigbee 2006 protocol stack are used as a software development platform, and the software process of detection node and monitoring gateway are designed. The design of monitoring sub-station is similar to the design of the monitoring gateway. Readers tend to read the references on the software process of surface monitoring center.

The wireless network gateway, as the network coordinator, starts to create a wireless network by sending periodically beacon after powering. The monitoring gateway starts receiving data information after network is created, and take appropriate action followed the fact that the data is judged and processed. The monitoring gateway's structure is shown in Fig. 4. 


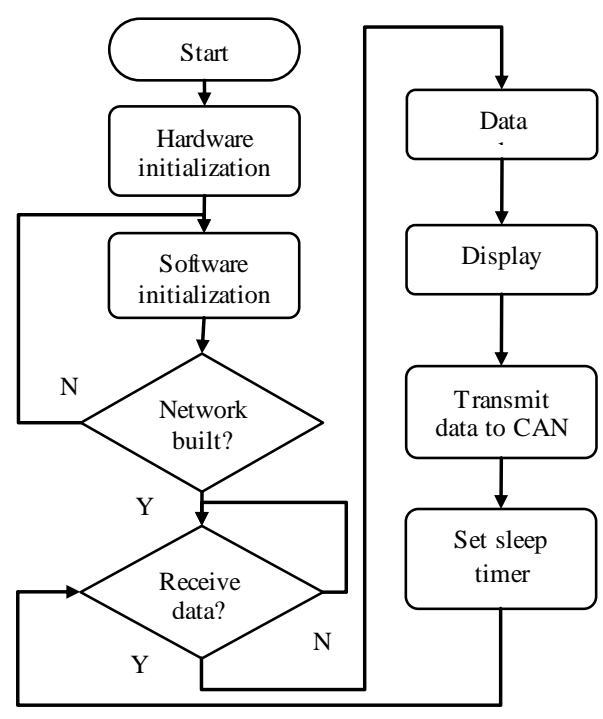

Figure 4. The flowchart of wireless gateway

The inspecting sensor nodes, as the detection terminals, begin to inspecting network after powering up and complete all initialization tasks. It will request to join the network when the routers are found, and start to take appropriate action to control. The mobile inspecting nodes begin to determine the network connection status when the control process is completed. If problems are occurred in the network connection, the appropriate testing and reconnect are made. The mobile detection node's specific processes are shown in Fig. 5.

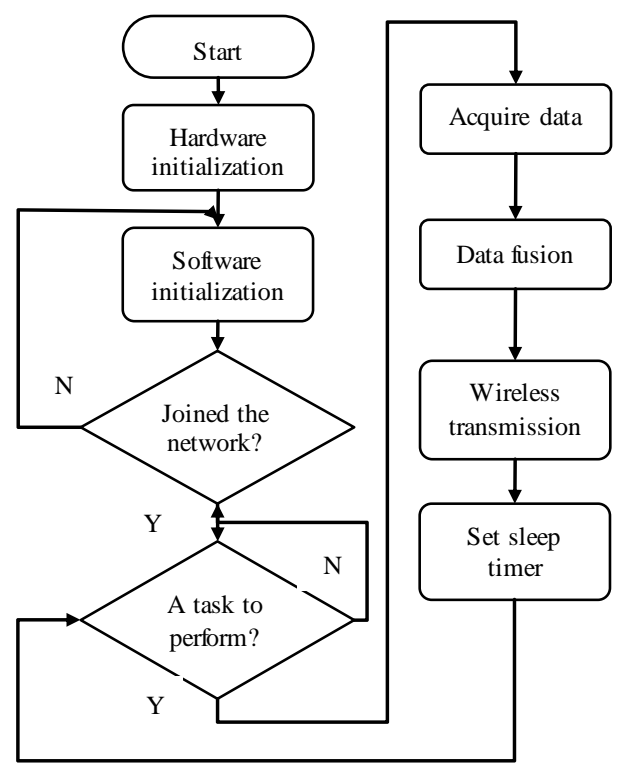

Figure 5. The flowchart of inspecting sensor node

\section{CONCLUSION}

A new mine gas inspecting system was described; it consists of multi-sensor, inspecting sensor nodes, substations and monitoring centre, which is a wireless selforganized network communication protocol that has lots of advantages including low power consumption, low cost, short delay and high capacity. This system can not only detect the gas concentration, temperature and relative humidity, but also can make data fusion to forecast the potential hazard so as to reduce loss of life and property. This system can be flexibly deployed and it overcomes the shortcomings of wire systems because of the technology of WSN. As a result, it can offset the weaknesses of current systems. Mobile inspecting nodes in system deployed in miner's lamps can locate its positions, while deployed in hand-held gas inspectors can inspect gas concentration to avoid the incomplete detection and missed detection. The technology of WSN strengthens flexibility, maintainability and extendibility of coal mine gas inspecting system. Furthermore, the system modulation and the open style structure enable the system to have a good probability. Above all, WSN and technology of data fusion used in coal mine inspecting system are attracting technology, which can make the field production more safely. This systemhas great promising future.

\section{ACKNOWLEDGMENT}

In this paper, the research was sponsored by the National Natural Science Foundation of China (61172127) and the 211 Project of Anhui University (KJQN1121) and the National Undergraduate Innovative Training Program (J18511044) and Innovative Training Program of Anhui province (201510357230).

\section{REFERENCES}

[1] Xianli Q, Mingchao F, Bin S. Coal mine gas wireless monitoring system based on WSNs[C]//Digital Manufacturing and Automation (ICDMA), 2011 Second International Conference on. IEEE, 2011: 309-312.

[2] Dongxuan Y, Yan C, Kedong W. Design of environmental monitoring node of coal mine based on CC2530[C]//Computer Application and System Modeling (ICCASM), 2010 International Conference on. IEEE, 2010, 14: V14-418-V14-421.

[3] Wang Jian, Wang Rulin. Coal mine gas monit oring wireless sensor network system's design and Implement[J]. Journal of China University of Mining and Technology. 2006, 10: 112-114. (in Chinese)

[4] Gaoa Z, Huangb L. A Forest Fire Monitoring and Early Warning System Based on the Technology of Multi-sensor and Multilevel Data Fusion[J]. 2015

[5] Dohare Y S, Maity T, Paul P S, et al. Design of surveillance and safety system for underground coal mines based on low power WSN[C]//Signal Propagation and Computer Technology (ICSPCT), 2014 International Conference on. IEEE, 2014: 116-119. 